\title{
Airway Management for Treacher Collins Syndrome with Limited Mouth Opening
}

\author{
Aiji Boku*, Hiroshi Hanamoto, Chiho Kudo, Yoshinari Morimoto, Mistutaka Sugimura, \\ Midori Tooyama, Hitoshi Niwa
}

Department of Dental Anesthesiology, Graduate School of Dentistry, Osaka University, Suita, Japan.

Email: "bokuaiji@dent.osaka-u.ac.jp, Hanamoto@dent.osaka-u.ac.jp, ckudo@dent.osaka-u.ac.jp, ysn-mori@dent.osaka-u.ac.jp, smit@dent.osaka-u.ac.jp, tooyama@dent.osaka-u.ac.jp, niwa@dent.osaka-u.ac.jp

Received January $20^{\text {th }}, 2013$; revised February $25^{\text {th }}, 2013$; accepted March $8^{\text {th }}, 2013$

\begin{abstract}
Patients with Treacher Collins Syndrome (TCS) present unique airway management problems for anesthesiologists due to mandibular micrognathia, the small oral aperture, and temporomandibular joint anomalies. We describe the case of a pediatric TCS patient with limited mouth opening who experienced severe airway obstruction during deep inhalation anesthesia (sevoflurane following i.v. midazolam) for routine dental work. When difficult airway management is expected, intubation of conscious patients is a well-recognized technique in adults; however, it is rarely appropriate for pediatric patients who usually do not cooperate. According to general anesthesia algorithms for pediatric patients with difficult airways, in most pediatric patients, tracheal intubation is performed after the induction of general anesthesia and some authors have reported the usefulness of LMA for maintaining airway patency in patients with TCS. However, in our case LMA could not be used because of severe limitation of mouth opening. In addition, the LMA is so bulky that it is impossible to insert a LMA into patients with narrow airway anatomy. We initially planned to carry out fiberoptic intubation while awake and under sedation if the airway patency could not be secured after the induction of anesthesia. The patient was sedated properly with midazolam and sevoflurane, and awake fiberoptic intubation was performed uneventfully. Our experience in this case highlighted that careful planning of backup contingencies is important in achieving fiberoptic intubation and maintaining airway patency in pediatric TCS patients with limited mouth opening, and that awake intubation can be successful even in pediatric patients.
\end{abstract}

Keywords: Treacher Collins Syndrome; Airway Management; Awake Intubation; Micrognathia

\section{Introduction}

Treacher Collins Syndrome (TCS) is a congenital genetic anomaly arising from defective growth of the first branchial arch [1] and an onset incidence is about one person per 10,000 people. TCS is known to be associated with upper airway obstruction and difficult intubation during airway management for anesthesia because of mandibular micrognathia, a small oral aperture, and a temporomandibular joint abnormality. It has been suggested that intubation of patients with TCS becomes more difficult with increasing age [2]. We present a pediatric patient with TCS in whom sedated awake fiberoptic tracheal intubation was successful.

\section{Case Report}

An 11-year-old girl (height: $129 \mathrm{~cm}$, weight: $23 \mathrm{~kg}$ ) was scheduled for dental treatment under general anesthesia

\footnotetext{
*Corresponding author.
}

due to multiple dental caries and extreme dental phobia. She had a history of difficult airway since birth. She had undergone elective lip-tongue adhesion under general anesthesia at the age of 1 month. When she was 1 year old, the release of the fixed tongue was performed under general anesthesia, and her mother reported that difficult intubation had been encountered. The patient could not sleep in a supine position without a nasopharyngeal airway until age 6 . She had severe mandibular micrognathia with limitation of mouth opening, and her inter-incisor distance was less than $1 \mathrm{~cm}$ (Figures 1 and 2).

She was given atropine sulfate intramuscularly as a premedication. After standard monitoring was applied in the operating room, an intravenous line was placed and midazolam $2 \mathrm{mg}$ was administered in three divided doses because she was slightly excited. General anesthesia was induced by sevoflurane in oxygen with a stepwise increase of inhaled concentration via an endoscopic mask. This mask has a hole for the insertion of fiberscope and enables us to carry out fiberoptic intubation while maintaining 




Figure 1. She had severe mandibular micrognathia.

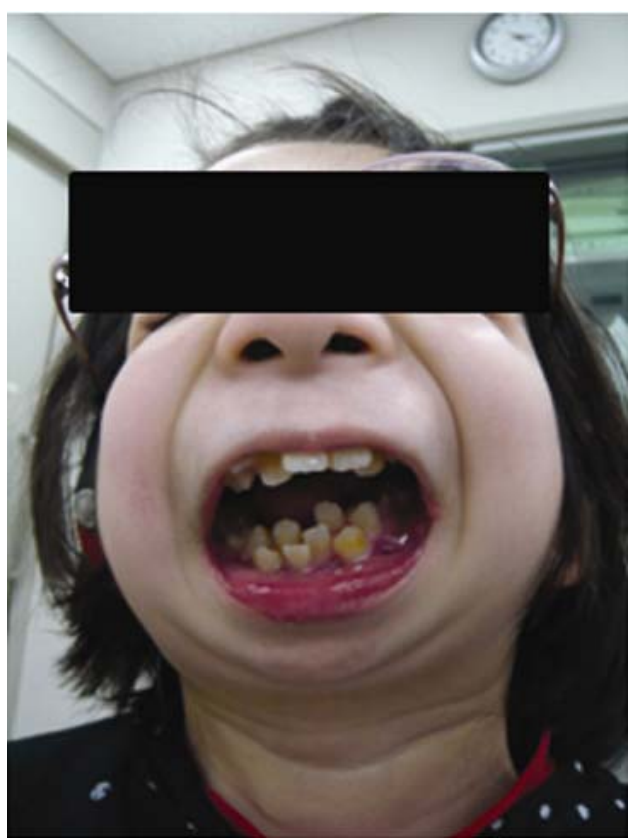

Figure 2. Inter-incisor distance was less than $1 \mathrm{~cm}$.

mask ventilation.

Although spontaneous ventilation was maintained, inadequate chest movement due to nearly complete airway obstruction was observed with an increase in the depth of anesthesia. Because a two-handed facemask hold including a jaw thrust, mouth opening, and chin lift maneuver failed to secure patency of the airway, a nasopharyngeal airway was inserted. However, it had only a limited effect on the improvement of the airway patency. Hence, we decided to turn off inhalational anesthetics and wake the patient up to avoid putting her at greater risk of a "cannot ventilate, cannot intubate" situation. As the depth of anesthesia decreased, adequate assisted ventilation became possible again. Although she did not respond to verbal stimulation, she moved her extremities slightly. She was considered to be under sedation with midazolam and sevoflurane. At that time, a 1.8-mm outer diameter fiberscope (Olympus LE-P: Olympus, Tokyo, Japan) was inserted through the small hole in the endoscopic mask. Nasal tracheal intubation with a 5.0 cuffed endotracheal tube (Ivory Portex Blue Line) was successfully performed without difficulty (Figure 3), and propofol $30 \mathrm{mg}$ was administered. The patient subsequently suffered no laryngospasm, coughing, breath holding, or desaturation. After the completion of the operation, the endotracheal tube was removed after she became fully awake. The respiratory state after extubation was normal.

\section{Discussion}

When difficult airway management is expected, intubation of conscious patients is a well-recognized technique in adults; however, it is rarely appropriate for pediatric patients who usually do not cooperate. General anesthesia algorithms for pediatric patients with difficult airways have been published [3]. According to these guidelines, in most pediatric patients, tracheal intubation is performed after the induction of general anesthesia. Many

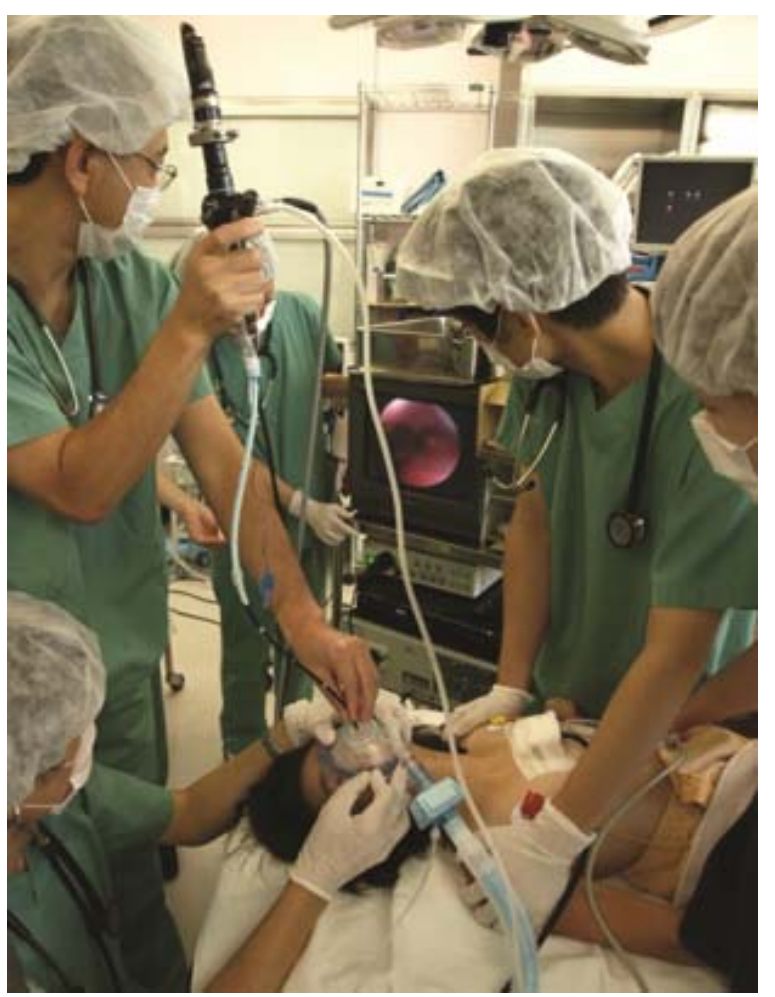

Figure 3. Awake fiberoptic tracheal intubation via an endoscopic mask. 
anesthesiologists prefer inhalational induction with sevoflurane, and intubation is performed while maintaining spontaneous respiration. However, airway obstruction often develops with an increase in the depth of anesthesia $[1,4]$. A triple airway maneuver and insertion of an oral or nasal airway are used to relieve airway obstruction. If these methods are unsuccessful, the laryngeal mask airway (LMA) is the next step to secure the patency of the airway. Fiberoptic tracheal intubation can be performed through a LMA, and some authors have reported the usefulness of LMA for maintaining airway patency in patients with TCS [2,5]. On the other hand, Sophie et al. reported the usefulness of GlideScope for tracheal intubation in patient with TCS [6]. However, in our case LMA or GlideScope could not be used because of severe limitation of mouth opening. In addition, the LMA is so bulky that it is impossible to insert a LMA into patients with narrow airway anatomy. Therefore, the only remaining option was to wake up the patient. However, the pediatric difficult airway algorithm does not specify the next step after waking up the patient. We initially planned to carry out fiberoptic intubation while awake and under sedation if the airway patency could not be secured after the induction of anesthesia. The patient was sedated properly with midazolam and sevoflurane, and awake fiberoptic intubation was performed uneventfully.

\section{Conclusion}

Our experience in this case highlighted that careful planning of backup contingencies is important in achieving fiberoptic intubation and maintaining airway patency in pediatric TCS patients with limited mouth opening, and that awake intubation can be successful even in pediatric patients.

\section{REFERENCES}

[1] T. C. Lin, L. Y. Soo, T. I. Chen, I. C. Lu and H. T. Hsu, "Perioperative Airway Management in a Child with Treacher Collins Syndrome,” Acta Anaesthesiologica Taiwanica, Vol. 47, No. 1, 2009, pp. 44-47. doi:10.1016/S1875-4597(09)60021-7

[2] J. Hosking, D. Zoanetti, A. Carlyle, P. Anderson and D. Costi, "Anesthesia for Treacher Collins Syndrome: A Review of Airway Management in 240 Pediatric Cases," Anesthesia \& Pain Management, Vol. 22, No. 8, 2012, pp. 752-758. doi:10.1111/j.1460-9592.2012.03829.x

[3] K. Crocker and A. E. Black, “Assessment and Management of the Predicted Difficult Airway in Babies and Children,” Anaesthesia \& Intensive Care Medicine, Vol. 10, No. 4, 2009, pp. 200-205. doi:10.1016/j.mpaic.2009.01.012

[4] F. S. Xue, Y. M. Zhang, X. Liao and J. H. Liu, “Airway Management for Pediatric Patients with Difficult Airways Due to Craniofacial Abnormalities," Anesthesia \& Pain Management, Vol. 19, No. 7, 2009, pp. 701-702. doi:10.1111/j.1460-9592.2009.02985.x

[5] W. L. McNiece and S. F. Dierdorf, "The Pediatric Airway,” Seminars in Pediatric Surgery, Vol. 13, No. 3, 2004, pp. 152-165.

doi:10.1053/j.sempedsurg.2004.04.008

[6] S. Bishop, P. Clements, K. Kale and M. R. Tremlett, "Use of GlideScope Ranger in the Management of a Child with Treacher Collins Syndrome in a Developing World Setting," Anesthesia \& Pain Management, Vol. 19, No. 7, 2009, pp. 695-696. doi:10.1111/j.1460-9592.2009.03021.x 